\title{
Oriented Hypergraphs: Introduction and Balance
}

\author{
Lucas J. Rusnak \\ Department of Mathematics \\ Texas State University \\ San Marcos, Texas, U.S.A. \\ lucas.rusnak@txstate.edu
}

\begin{abstract}
Submitted: Oct 1, 2012; Accepted: Sep 13, 2013; Published: Sep 26, 2013
Mathematics Subject Classifications: 05C22, 05C65, 05C75
\end{abstract}

\begin{abstract}
An oriented hypergraph is an oriented incidence structure that extends the concept of a signed graph. We introduce hypergraphic structures and techniques central to the extension of the circuit classification of signed graphs to oriented hypergraphs. Oriented hypergraphs are further decomposed into three families - balanced, balanceable, and unbalanceable - and we obtain a complete classification of the balanced circuits of oriented hypergraphs.
\end{abstract}

Keywords: Oriented hypergraph; balanced hypergraph; balanced matrix; signed hypergraph

\section{Introduction}

Oriented hypergraphs have recently appeared in [8] as an extension of the signed graphic incidence, adjacency, and Laplacian matrices to examine walk counting and provide a combinatorial model for matrix algebra. It is known that the cycle space of a graph is characterized by the dependent columns of its oriented incidence matrix — we further expand the theory of oriented hypergraphs by examining the extension of the cycle space of a graph to oriented hypergraphs.

In this paper we divide the problem of structurally classifying minimally dependent columns of incidence matrices into three categories - balanced, balanceable, and unbalanceable - and introduce new hypergraphic structures to obtain a classification of the minimal dependencies of balanced oriented hypergraphs. The balanceable and unbalanceable cases will be addressed in future papers.

The concept of a balanced matrix plays an important role in combinatorial optimization and integral programming, for a proper introduction see $[3,4,10,14]$. While the 
more general concept of a balanced incidence-oriented hypergraph was introduced in 1992 by C.J. Shi and extends the concept of a balanced matrix using signed graphs and has applications in VLSI design, integrated circuits, and logic synthesis [11, 12].

Incidence-oriented hypergraphs and balance were independently developed by Rusnak in [9] as a combinatorial model to extend algebraic and spectral graph theoretic results to integral matrices as well as examine the circuit structure of representable matroids - this paper is an adaptation of the introduction and classification of those balanced minimal dependency results.

The set of minimal dependencies, or circuits, provide a characterization of the vector matroid through the matroid circuit axioms (see [7]). We seek a hypergraphic model of the circuits of vector matroids that is an analog to the following known theorem:

Theorem 1.0.1. Let $G$ be a graph whose oriented incidence matrix is $\mathrm{H}$. The following are equivalent:

1. $E(C)$ is an edge set of a circuit of $G$,

2. $E(C)$ is a circuit in the graphic matroid $M(G)$,

3. the columns of $\mathrm{H}$ corresponding to $E(C)$ are minimally dependent.

The classification of oriented hypergraphic circuits will be done by examining the structure of the incidence matrix over the rationals to provide a way to apply graphic and signed graphic techniques to hypergraphs, as well as an alternate way to study and model representable matroids.

The family of balanced oriented hypergraphs contains graphs, so a characterization of the balanced circuits of oriented hypergraphs can be regarded as an extension of Theorem 1.0.1. The development of hypergraphic incidence orientation is a direct extension of the work by Zaslavsky in $[15,16,17]$, while the concept of balance is a relaxed version of the concepts which appear in $[1,2,4,13]$.

Section 2 introduces basic oriented hypergraphic definitions and the incidence matrix. Section 3 collects the operations relevant to the classification of oriented hypergraphic circuits. Section 4 discusses hypergraphic extensions of paths and cycles. Section 5 introduces the hypergraphic cyclomatic number, the incidence graph, and balance. These lead to the complete classification of the balanced oriented hypergraphic circuits in Section 6 .

\subsection{Signed Graphs}

A signed graph is a generalization of a graph that allows edges incident to 2 or fewer vertices and signs every 2-edge + or - . The edges incident to zero vertices are called loose edges, while the edges incident to exactly 1 vertex are called half edges. A circle of a signed graph is the edge set of a simple cycle, a half edge, or a loose edge. A circle is positive or negative according to the product of the signs of its edges. A loose edge is regarded as positive, while a half edge is regarded as negative. 
We say a signed graph is balanced if every circle is positive. The first characterization of balance was due to Harary [6]:

Theorem 1.1.1. A signed graph is balanced if, and only if, its vertices can be partitioned into sets $V_{1}$ and $V_{2}$ so that all negative edges have one end-point in $V_{1}$ and one end-point in $V_{2}$.

Harary continues to explain that this Theorem has the social interpretation of "two highly cohesive cliques which dislike each other."

A handcuff is a pair of disjoint circles connected by a path of length $\geqslant 1$, or two circles who only share a single vertex. If both circles of a handcuff are positive we say the handcuff balanced, and if both circles are negative we say the handcuff is contra-balanced.

While Zaslavsky introduces two natural matroids associated to a signed graph, our focus is on the frame matroid, which most faithfully extends the concepts of graph theory via the signed incidence matrix. The circuits of the signed graphic frame matroid are classified by the following theorem of Zaslavsky in [15].

Theorem 1.1.2. Let $\Sigma$ be a signed graph whose oriented incidence matrix is $\mathrm{H}$. The following are equivalent:

1. $E(C)$ is a positive circle or a contra-balanced handcuff in the signed graph $\Sigma$,

2. $E(C)$ is a circuit in the frame matroid $M(\Sigma)$,

3. the columns of $\mathrm{H}$ corresponding to $E(C)$ are minimally dependent.

We can regard a graph as a signed graph in which each edge is positive, so every circle of a graph is necessarily positive, thus Theorem 1.1.2 subsumes the graphic circuit classification. A complete classification of the matroid circuits provides the entire matroid structure - including bases, rank and closure - providing an alternate structural characterization for vector matroids.

\section{An Introduction to Oriented Hypergraphs}

\subsection{Introductory Definitions}

Let $V$ and $E$ be disjoint finite sets whose respective elements are called vertices and edges. An incidence function is a function $\iota: V \times E \rightarrow \mathbb{Z}_{\geqslant 0}$, while a vertex $v$ and an edge $e$ are said to be incident with respect to $\iota$ if $\iota(v, e) \neq 0$. The value $\iota(v, e)$ is called the multiplicity of incidence class $(v, e)$. Let $L(v, e, \iota)$ be a set of $\iota(v, e)$ distinct elements, called the labelling set of $(v, e)$. An incidence is a triple $(v, e, k)$ where $v$ and $e$ are incident and $k \in L(v, e, \iota)$.

Let $\mathcal{I}_{(\iota, \mathcal{L})}$ be the set of incidences determined by incidence function $\iota$ and labelling sets $\mathcal{L}$. Since the set $\mathcal{I}_{(\iota, \mathcal{L})}$ also determines the incidence function and labelling sets we immediately drop the subscript notation and simply write $\mathcal{I}$. An incidence orientation is a 
function $\sigma: \mathcal{I} \rightarrow\{+1,-1\}$. Every incidence $(v, e, k)$ is naturally extended to a quadruple $(v, e, k, \sigma(v, e, k))$ called an oriented incidence. An oriented hypergraph is the quadruple $(V, E, \mathcal{I}, \sigma)$. This formulation of oriented incidence is an extension of orientations of signed graphs in [17].

When drawing oriented hypergraphs the vertices are depicted as points in the plane while edges will be depicted as shaded regions in the plane whose incident vertices appear on its boundary. An oriented incidence $(v, e, k, \sigma(v, e, k))$ is drawn within edge $e$ as an arrow entering $v$ if $\sigma(v, e, k)=+1$, or an arrow exiting $v$ if $\sigma(v, e, k)=-1$.

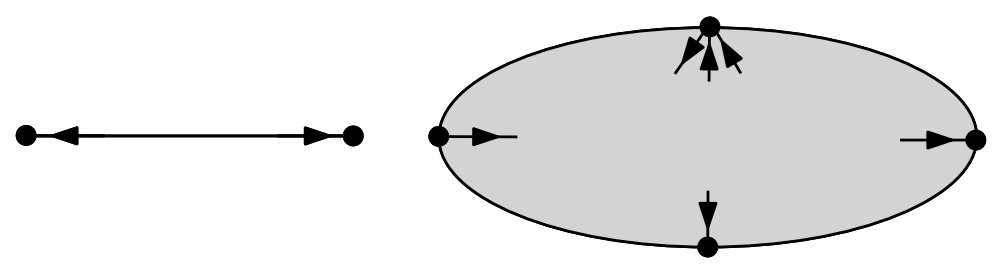

Figure 1: Two oriented hyperedges.

A triple $(V, E, \mathcal{I})$ is a hypergraph, and all definitions that do not depend on $\sigma$ will be defined on the underlying hypergraph of an oriented hypergraph and will be inherited by the oriented hypergraph.

A hypergraph is incidence-simple if $\iota(v, e) \leqslant 1$ for all $v$ and $e$, and for convenience we will write $(v, e)$ if $G$ is an incidence-simple hypergraph. Two, not necessarily distinct, vertices $v$ and $w$ are adjacent with respect to edge $e$ if there exists incidences $\left(v, e, k_{1}\right)$ and $\left(w, e, k_{2}\right)$ such that $\left(v, e, k_{1}\right) \neq\left(w, e, k_{2}\right)$. An adjacency is a quintuple $\left(v, k_{1} ; w, k_{2} ; e\right)$ where $v$ and $w$ are adjacent with respect to edge $e$ using incidences $\left(v, e, k_{1}\right)$ and $\left(w, e, k_{2}\right)$.

The degree, or valency, of a vertex is equal to the number of incidences containing that vertex and is denoted $\operatorname{deg}(v)$. A vertex whose degree equals 0 is isolated and a vertex whose degree equals 1 is monovalent. The size of an edge is the number of incidences containing that edge, and an edge of size $k$ is called a $k$-edge.

A path is a set of vertices, edges, and incidences of a hypergraph that form a sequence $a_{0}, i_{1}, a_{1}, i_{2}, a_{2}, i_{3}, a_{3}, \ldots, a_{m-1}, i_{m}, a_{m}$, where $\left\{a_{j}\right\}$ is an alternating sequence of vertices and edges, $i_{j}$ is an incidence containing $a_{j-1}$ and $a_{j}$, and no vertex, edge, or incidence is repeated. The first and last elements of this sequence are the end-points of the path. A path where both end-points are vertices is a vertex-path, a path where both end-points are edges is an edge-path, and a path where one end-point is a vertex and the other is an edge is a cross-path as it "crosses" the incidence structure from a vertex to an edge.

A hypergraph is connected if for any two distinct elements of $V \cup E$ there exists a path in $G$ containing them. A hypergraph that is not connected is disconnected. A connected component is a maximal connected subhypergraph. An edge whose set deletion from $E$ increases the number of connected components is an isthmus.

A circle of length $k$ is a set of $k$ vertices, $k$ edges, and $2 k$ incidences that form a sequence $a_{0}, i_{1}, a_{1}, i_{2}, a_{2}, i_{3}, a_{3}, \ldots, a_{2 k-1}, i_{2 k}, a_{2 k}$, where $\left\{a_{j}\right\}$ is an alternating sequence of vertices and edges, $i_{j}$ is an incidence containing $a_{j-1}$ and $a_{j}$, and no vertex, edge, or 
incidence is repeated except $a_{0}=a_{2 k}$. By symmetry we may assume that $a_{0}$ is a vertex. A circle $C$ is degenerate if, for some edge $a_{i} \in C$, there is a vertex $v \in C$ such that $v$ is not $a_{i-1}$ or $a_{i+1}$, and $v$ is incident to $a_{i}$. A circle that is not degenerate is called pure.

Given a hypergraph $G$ and a monovalent vertex $v$ of $G$ we say $v$ is a leaf of $G$ if the edge incident to $v$ is not contained in a circle of $G$, however, we say $v$ is a thorn of $G$ if the edge incident to $v$ is contained in some circle of $G$.

Given an adjacency $\left(v, k_{1} ; w, k_{2} ; e\right)$ we define the sign of the adjacency as

$$
\operatorname{sgn}_{e}\left(v, k_{1} ; w, k_{2}\right)=-\sigma\left(v, e, k_{1}\right) \sigma\left(w, e, k_{2}\right) .
$$

This is shortened to $\operatorname{sgn}_{e}(v, w)=-\sigma(v, e) \sigma(w, e)$ if $G$ is incidence-simple. If $v$ and $w$ are not adjacent via edge $e$ we say the sign of the non-adjacency is 0 . It should be noted that signed 2-edges as discussed in [15] correspond to an edge with a single signed adjacency for oriented hypergraphs.

If $B=\left\{a_{0}, i_{1}, a_{1}, i_{2}, a_{2}, i_{3}, a_{3}, \ldots, a_{n-1}, i_{n}, a_{n}\right\}$ is a circle or a path, then the sign of $B$ is

$$
\operatorname{sgn}(B)=(-1)^{p} \prod_{h=1}^{n} \sigma\left(i_{h}\right),
$$

where

$$
p=\left\lfloor\frac{n}{2}\right\rfloor .
$$

This implies that the sign of a circle is the product of the signs of all adjacencies in the circle.

Since Theorems 1.0.1 and 1.1.2 examine circle structures to characterize their matroid circuits it is natural to examine hypergraphic circle structure. However, it is necessary to regarded each incidence as unique since two circles may differ by a single incidence and have different signs even though they have the same vertex and edge set - a configuration that cannot exist in graphs and signed graphs.

Given a hypergraph $G=\left(V_{G}, E_{G}, \mathcal{I}_{G}\right)$ a subhypergraph $H$ of $G$ is the hypergraph $H=\left(V_{H}, E_{H}, \mathcal{I}_{H}\right)$ where $V_{H} \subseteq V_{G}, E_{H} \subseteq E_{G}$, and $\mathcal{I}_{H} \subseteq \mathcal{I}_{G} \cap\left(V_{H} \times E_{H} \times \mathbb{Z}\right)$. This definition is more relaxed than conventional definitions as it allows for only parts of edges to appear in the subhypergraph, giving the flexibility to have incidence-centric treatments of subhypergraphs in addition to the usual edge-centric and vertex-centric subhypergraphs.

We are often interested in subhypergraphs with more structure than a general subhypergraph. Let $G=(V, E, \mathcal{I})$ be a hypergraph, and let $U \subseteq V$ and $F \subseteq E$. The cross-induced subhypergraph of $G$ on $(U, F)$ is the subhypergraph $G:(U, F)=(U, F, \mathcal{I} \cap$ $(U \times F \times \mathbb{Z}))$. If $U=V$ we say that the subhypergraph is an edge-restriction to $F$ and write $G \mid F$. An edge-induced hypergraph is the hypergraph $G: F=(W, F, \mathcal{I} \cap(W \times F \times \mathbb{Z}))$ where $W=\{v \in V: v$ is incident to some $f \in F\}$. All hypergraphic containment will take place in the edge-induced ordering unless otherwise stated. 


\subsection{The Incidence Matrix}

Given a labeling $v_{1}, v_{2}, v_{3}, \ldots, v_{m}$ of the elements of $V$, and $e_{1}, e_{2}, e_{3}, \ldots, e_{n}$ of the elements of $E$, of an oriented hypergraph $G$, the incidence matrix of $G$ is the $m \times n$ matrix $\mathrm{H}_{G}=\left[\eta_{i j}\right]$, where

$$
\eta_{i j}=\sum_{k=1}^{\iota\left(v_{i}, e_{j}\right)} \sigma\left(v_{i}, e_{j}, k\right) .
$$

If $G$ is incidence-simple, then this is equivalent to

$$
\eta_{i j}= \begin{cases}0, & \text { if }\left(v_{i}, e_{j}\right) \notin \mathcal{I}, \\ 1, & \text { if } \sigma\left(v_{i}, e_{j}\right)=+1, \\ -1, & \text { if } \sigma\left(v_{i}, e_{j}\right)=-1 .\end{cases}
$$

Every incidence-simple oriented hypergraph with a labeled vertex set and labeled edge set has a representation as a $\{0, \pm 1\}$-matrix using its incidence matrix. Moreover, a $\{0, \pm 1\}$-matrix with labeled columns and rows has a unique representation as an incidencesimple oriented hypergraph with edge set equal to the column labels, vertex set equal to the row labels, and a vertex $v$ and an edge $e$ are incident if the $(v, e)$-entry in the matrix is non-zero.

Non-incidence-simple oriented hypergraphs may have incidence matrix entries other than $0,+1$, or -1 , for example, if there are $n$ incidences containing the same vertex and edge each oriented +1 , then a value of $+n$ would appear in the incidence matrix, for this reason we consider our matrices over the rationals. It is also possible that two incidences at the same vertex within the same edge could be signed +1 and -1 and produce a net value of 0 in the incidence matrix. To avoid such ambiguities all multiple incidences are regarded as having the same orientation unless stated otherwise.

An oriented hypergraph is said to be dependent if the columns of its incidence matrix are dependent, and adopt similar conventions for all matrix related terminology. The classification of the minimal column dependencies of a $\{0, \pm 1\}$-matrix $\mathrm{H}$ begins with the following simple lemma.

Lemma 2.2.1. If an oriented hypergraph contains a monovalent vertex, then it is not minimally dependent.

Proof. If an oriented hypergraph contains a monovalent vertex, then there is a row with a single non-zero entry and the corresponding column cannot belong to a minimal dependency.

\subsection{Incidence Duality}

Given a hypergraph $G$ the incidence dual $G^{*}$ is the hypergraph obtained by reversing the roles of the vertices and edges. That is, given an oriented hypergraph $G=(V, E, \mathcal{I}, \sigma)$, its incidence dual $G^{*}$ is the oriented hypergraph $\left(E, V, \mathcal{I}^{*}, \sigma^{*}\right)$ where $\mathcal{I}^{*}=\{(e, v, k)$ : $(v, e, k) \in \mathcal{I}\}$, and $\sigma^{*}: \mathcal{I}^{*} \rightarrow\{+1,-1\}$ such that $\sigma^{*}(e, v, k)=\sigma(v, e, k)$. Observe that 
$\mathcal{I}^{*}$ determines an incidence function $\iota^{*}$ where $\iota^{*}(e, v)=\iota(v, e)$. In graph theory a line graph can be regarded as the graphical approximation of incidence duality. A number of algebraic graph theoretic results hold in the more general setting of oriented hypergraphs and incidence duality, see [8].

A number of structures are closed under incidence duality. By interchanging the roles of edges and vertices the incidence dual of a path is still a path. Specifically, the incidence dual of a vertex-path is an edge-path, the incidence dual of an edge-path is a vertex-path, and the incidence dual of a cross-path is a cross-path. Similarly, the incidence dual of a circle is still a circle. However, we have a better result for circles:

Lemma 2.3.1. The following are true for a circle $C$ in an oriented hypergraph $G$.

1. $C$ is pure in $G$ if, and only if, $C^{*}$ is pure in $G^{*}$.

2. The sign of $C$ in $G$ is equal to the sign of $C^{*}$ in $G^{*}$.

Proof. Incidence duality reverses vertices and edges, which, by symmetry, does not alter purity of a circle. Moreover, the incidence signs are also unchanged in the incidence dual, so the sign of a circle also remains unchanged.

\section{Operations on Oriented Hypergraphs}

\subsection{Deletion, Switching, and 2-Contraction}

Weak edge-deletion of edge $e$, denoted $G \backslash e$, is the hypergraph resulting from the set deletion of the edge $e$ from $E$ along with the removal of any incidences containing $e$ from $\mathcal{I}$. The incidence dual of weak edge-deletion is weak vertex-deletion and is denoted $G \backslash v$ for $v \in V$, and removes the vertex $v$ from $V$ along with any incidences containing $v$. The removal of a single edge or a single vertex has the following effect on the incidence matrix.

Lemma 3.1.1. Weak edge-deletion and weak vertex-deletion are equivalent to columndeletion and row-deletion in the corresponding incidence matrix.

Deletion of a vertex along with all incident edges is called strong vertex-deletion, while its incidence dual operation is strong edge-deletion.

A vertex-switching function is any function $\theta: V \rightarrow\{-1,+1\}$. Vertex-switching the oriented hypergraph $G$ means replacing $\sigma$ by $\sigma^{\theta}$, defined by: $\sigma^{\theta}\left(v, e, k_{1}\right)=\theta(v) \sigma\left(v, e, k_{1}\right)$; producing the oriented hypergraph $G^{\theta}=\left(V, E, \mathcal{I}, \sigma^{\theta}\right)$. Vertex-switching produces an adjacency sign $\operatorname{sgn}^{\theta}$, defined by: $\operatorname{sgn}_{e}^{\theta}\left(v, k_{1} ; w, k_{2}\right)=\theta(v) \operatorname{sgn}_{e}\left(v, k_{1} ; w, k_{2}\right) \theta(w)$.

Edge-switching is the incidence dual of vertex-switching, and negates all incidences that contain a given edge.

Lemma 3.1.2. Edge-switching and vertex-switching are equivalent to column negation or row negation, respectively, in the corresponding incidence matrix. 
Lemma 3.1.3. Edge-switching does not alter the signs of any adjacencies in an oriented hypergraph.

Proof. Consider the adjacency $\left(v, k_{1} ; w, k_{2} ; e\right)$. Since switching an edge $e$ negates all incidences containing $e$, the sign of this adjacency is

$$
\operatorname{sgn}_{e}\left(v, k_{1} ; w, k_{2}\right)=-\sigma\left(v, e, k_{1}\right) \sigma\left(w, e, k_{2}\right)
$$

before switching, and has sign

$$
-\left[-\sigma\left(v, e, k_{1}\right)\right]\left[-\sigma\left(w, e, k_{2}\right)\right]=\operatorname{sgn}_{e}\left(v, k_{1} ; w, k_{2}\right)
$$

after switching.

Lemma 3.1.4. Vertex-switching does not alter the signs of any circles in an oriented hypergraph.

Proof. Let $C=a_{0}, i_{1}, a_{1}, i_{2}, a_{2}, i_{3}, a_{3}, \ldots, a_{2 k-1}, i_{2 k}, a_{2 k}$ be a circle and $a_{j}$ is the vertex we wish to switch. Switching $a_{j}$ will negate incidences $i_{j}$ and $i_{j+1}$, and the switched circle will have the same sign.

Switching plays an essential part in defining contraction in an oriented hypergraph in order to have it agree with matroid contraction in the column dependency matroid of the incidence matrix. Because we will later restrict ourselves to a certain family of oriented hypergraphs, we only need to focus on the contraction of 2-edges and its incidence dual operation.

The origins of signed 2-edge-contraction appear in [15] and its development remains faithful to the corresponding matroidal contraction. A positive 2-edge is contracted as a graphic edge, while a negative 2-edge is contracted by first switching one of the incident vertices so that the edge is positive and then contracting the edge.

Incidence dual to 2-edge-contraction is 2-vertex-contraction and can be performed by taking the incidence dual, contracting the corresponding edge, and then dualizing again. Since there are only 2 incidences containing a contracted 2-edge, those same 2 incidences are the only incidences containing the dual vertex. We say a vertex is compatibly oriented (with respect to two of its incidences) if the product of the two incidences is negative. Compatible 2-vertex-contraction has the effect of combining the two incident edges into a single new edge with the contracted vertex removed.

Lemma 3.1.5. Let $G$ be a minimally dependent oriented hypergraph. If $G^{\prime}$ is obtained by a 2-vertex-contraction of $G$, then $G^{\prime}$ is minimally dependent.

Proof. Let $G=(V, E, \mathcal{I}, \sigma)$ be a minimally dependent oriented hypergraph where $V=$ $\left\{v_{1}, v_{2}, \ldots, v_{m}\right\}$ and $E=\left\{e_{1}, e_{2}, \ldots, e_{n}\right\}$. Let $v_{1}$ be the degree- 2 vertex we wish to contract, and suppose the edges are labeled so that $v_{1}$ is incident to edges $e_{1}$ and $e_{2}$. We may assume that $v_{1}$ is compatibly oriented, if it is not we can switch an incident edge since switching does not alter minimal dependencies. 
Since $G$ is minimally dependent, solving $\mathrm{Hx}=\mathbf{0}$ yields a fully supported coefficient vector $\mathbf{x}$. This corresponds to the linear system

$$
\sum_{i=1}^{n} \alpha_{i} \mathbf{e}_{i}=\mathbf{0}
$$

where the values $\alpha_{i}$ are the entries of $\mathbf{x}$. Summing only row $v_{1}$ we see that $\alpha_{1} e_{1,1}+\alpha_{2} e_{1,2}=$ 0 since $\operatorname{deg}\left(v_{1}\right)=2$. Since $v_{1}$ is compatible we know that if $e_{1,1}= \pm 1$, then $e_{1,2}=\mp 1$, so $\alpha_{1}=\alpha_{2}$. Thus columns $\mathbf{e}_{1}$ and $\mathbf{e}_{2}$ may be replaced with a single new column $\mathbf{e}=\mathbf{e}_{1}+\mathbf{e}_{2}$, and row $v_{1}$ may be deleted as it contains only 0 entries. The resulting oriented hypergraph remains minimally dependent.

\subsection{Subdivision and Column Splitting}

The inverse of 2-vertex-contraction is called edge-subdivision. In a drawing of an oriented hypergraph, edge-subdivision bipartitions the incidences of an edge and "pinches off" the edge to produce a new degree- 2 vertex between two newly created edges. A subdivision is compatible if the product of the two new incidences is negative and is incompatible if the product of the two new incidences is positive. If a subdivision is compatible we can immediately contract the newly introduced vertex to reclaim the original oriented hypergraph.

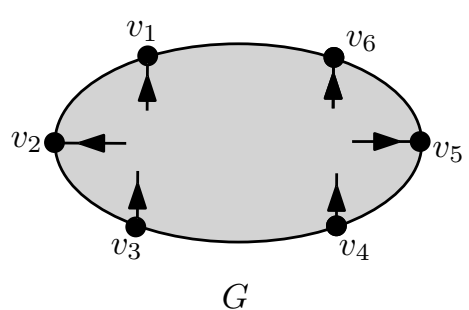

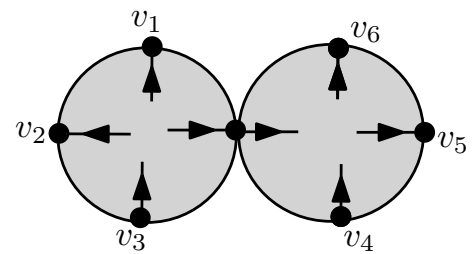

$G_{1}$

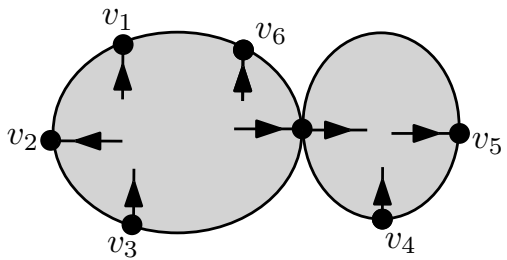

$G_{2}$

Figure 2: Two different subdivisions of a hyperedge.

Compatible subdivision plays a central role in understanding the structure of dependencies for two reasons. First, compatible subdivision does not alter the signs of any existing circles. Second, compatible subdivision does not alter minimal dependencies.

Lemma 3.2.1. The sign of a path between two vertices in any compatible subdivision of an edge $e$ is equal to the sign of their adjacency in $e$. 
Proof. Let $v$ and $w$ be two vertices incident to edge $e$, and edge $e$ is to be subdivided into edges $e_{1}$ and $e_{2}$. If $v$ and $w$ and in the same side of the bipartition of a subdivision of $e$ they will have the same adjacency sign. If $v$ and $w$ are in different parts of the bipartition of $e$ the newly introduced vertex $u$ between them is compatibly oriented and the sign of the resulting $v w$-path is

$$
\begin{aligned}
{\left[-\sigma\left(v, e_{1}\right) \sigma\left(u, e_{1}\right)\right]\left[-\sigma\left(u, e_{2}\right) \sigma\left(w, e_{2}\right)\right] } & =\left[\sigma\left(u, e_{1}\right) \sigma\left(u, e_{2}\right)\right]\left[\sigma\left(v, e_{1}\right) \sigma\left(w, e_{2}\right)\right] \\
& =-\left[\sigma\left(v, e_{1}\right) \sigma\left(w, e_{2}\right)\right] \\
& =-[\sigma(v, e) \sigma(w, e)] .
\end{aligned}
$$

Which is the same as the original adjacency sign in $e$.

From this and taking repeated compatible subdivisions we have the following corollary.

Corollary 3.2.2. Let $G$ be an oriented hypergraph, $G_{1}$ be obtained from $G$ by a finite number of compatible subdivisions, $C$ be a circle of $G$, and $C_{1}$ the corresponding subdivided circle of $C$ in $G_{1}$. The sign of $C$ in $G$ is equal to the sign of $C_{1}$ in $G_{1}$.

The operation of subdivision has an effect on the incidence matrix called column splitting. As with subdivision, we have compatible and incompatible column splitting depending on whether the associated subdivision was compatible or incompatible.

Lemma 3.2.3. Let $M$ be an $m \times n\{0, \pm 1\}$-matrix and $M^{\prime}$ be a matrix obtained by compatible column splitting $M$. If $M$ is minimally dependent, then so is $M^{\prime}$.

Proof. Since the columns of $M$ are minimally dependent there is a single solution (up to scaling) of the matrix equation $M \mathbf{x}=\mathbf{0}$. Moreover, by minimality, the vector $\mathbf{x}$ satisfying this equation must have full support as no smaller supported vector can produce a dependency. Writing this as a linear combination of the column vectors we have

$$
\sum_{i=1}^{n} \alpha_{i} \mathbf{c}_{i}=\mathbf{0}
$$

where the $\alpha_{i}$ 's are the entries of $\mathbf{x}$ and $\mathbf{c}_{i}$ is the column vector corresponding to column $i$.

Let $\mathbf{c}_{1}$ be the column split into the new columns $\mathbf{d}_{1}^{\prime}$ and $\mathbf{d}_{2}^{\prime}$, and the new row created be $\mathbf{r}$. We can assume that $\mathbf{r}$ is introduced as the last row of the newly formed matrix $M^{\prime}$. Let $\mathbf{d}_{1}$ and $\mathbf{d}_{2}$ be the column vectors obtained by removing row $\mathbf{r}$ from $\mathbf{d}_{1}^{\prime}$ and $\mathbf{d}_{2}^{\prime}$ respectively.

Extend each column $\mathbf{c}_{i}, i \geqslant 2$, to a new column $\mathbf{c}_{i}^{\prime}$ where

$$
\mathbf{c}_{i}^{\prime}=\left[\begin{array}{c}
\mathbf{c}_{i} \\
0
\end{array}\right]
$$

and the entry 0 appears in row $\mathbf{r}$. Thus the matrix $M^{\prime}$ obtained by this compatible column splitting is

$$
\begin{aligned}
M^{\prime} & =\left[\begin{array}{l|l|l|l|l|l}
\mathbf{d}_{1}^{\prime} & \mathbf{d}_{2}^{\prime} & \mathbf{c}_{2}^{\prime} & \mathbf{c}_{3}^{\prime}|\ldots| & \mathbf{c}_{n}^{\prime}
\end{array}\right] \\
& =\left[\begin{array}{l|l|l|l|l|l}
\mathbf{d}_{1} & \mathbf{d}_{2} & \mathbf{c}_{2} & \mathbf{c}_{3} & \ldots & \mathbf{c}_{n} \\
\pm 1 & \mp 1 & 0 & 0 & \ldots & 0
\end{array}\right]
\end{aligned}
$$


where the last row is row $\mathbf{r}$.

Taking the same $\alpha_{i}$ 's, $i \geqslant 2$, that determined the minimal dependency for $M$, we let the coefficients on both $\mathbf{d}_{1}^{\prime}$ and $\mathbf{d}_{2}^{\prime}$ be $\alpha_{1}$. Taking this linear combination of columns of $M^{\prime}$ gives us

$$
\sum_{i=2}^{n} \alpha_{i} \mathbf{c}_{i}^{\prime}+\alpha_{1} \mathbf{d}_{1}^{\prime}+\alpha_{1} \mathbf{d}_{2}^{\prime} .
$$

For rows $\mathbf{r}_{1}$ through $\mathbf{r}_{m}$ we necessarily get 0 as this corresponds to the original linear combination of columns except that we use either $\mathbf{d}_{1}$ or $\mathbf{d}_{2}$ depending on which column supports the entry of $\mathbf{c}_{1}$. However, for row $\mathbf{r}$ the linear system gives $\pm \alpha_{1} \mp \alpha_{1}=0$, so this linear combination forms a dependency.

The dependency is clearly minimal as both new columns are required in the dependency, and $\alpha_{1} \neq 0$ since the original matrix was minimally dependent and no $\alpha_{i}$ is zero.

Corollary 3.2.4. If $G$ is minimally dependent and $G^{\prime}$ is a compatible subdivision of $G$, then $G^{\prime}$ is minimally dependent.

\section{Circle and Path Analogs}

\subsection{Inseparability and Flowers}

So far we have only translated the simple, closed path, property of a graphic circle to oriented hypergraphs. We now extend another property of graphic circles: the property of being minimally inseparable.

An oriented hypergraph is inseparable if every pair of incidences is contained in a circle. An inseparable oriented hypergraph is circle-covered if it contains a circle, or is a single 0-edge. The concept of circle-covered prevents degenerate inseparable oriented hypergraphs from being considered, like a single 1-edge.

Lemma 4.1.1. A circle-covered hypergraph contains no monovalent vertices, 1-edges, or isolated vertices.

A flower is a circle-covered oriented hypergraph that is minimal in the edge-induced subhypergraphic ordering. Clearly every graphic circle is a flower, however, there are additional flowers in oriented hypergraphs other than circles.
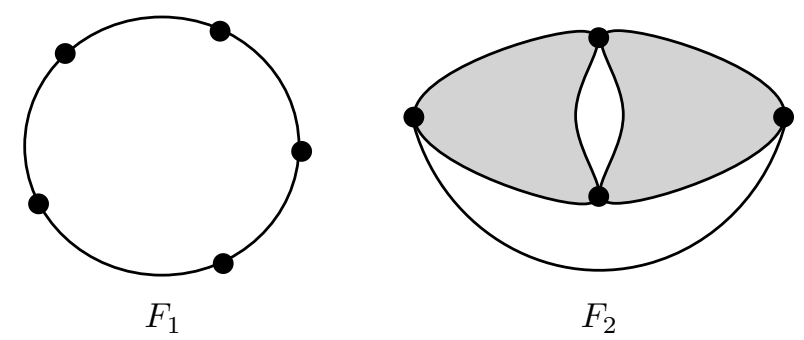

Figure 3: Two examples of flowers. 
While flowers of an oriented hypergraph can have many circles, the concept of a flower is simplified in signed graphs.

Proposition 4.1.2. $F$ is a flower of a signed graph if, and only if, $F$ is a circle or a loose edge.

A pseudo-flower is an oriented hypergraph containing at least one thorn such that the weak-deletion of all thorns results in a flower. The subhypergraph resulting from the weak-deletion of thorns in a pseudo-flower is called its flower-part. A $k$-pseudo-flower is a pseudo-flower with exactly $k$ thorns. Observe that a flower is inseparable so a pseudoflower is not a flower.
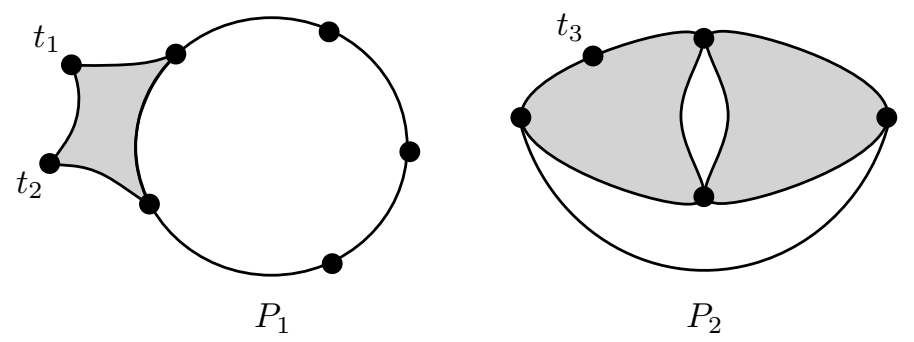

Figure 4: Two pseudo-flowers that contain the hypergraphs from Figure 3 as flower-parts.

Pseudo-flowers occur only as a degenerate example in signed graphs.

Proposition 4.1.3. $P$ is a pseudo-flower of a signed graph if, and only if, $P$ is a half edge.

\subsection{Arteries}

An artery is a connected, circle-free, 1-edge-free hypergraph in which the degree of every vertex is 1 or 2 , or is a single vertex. The divalent vertices of an artery are called internal vertices of the artery, while the non-divalent vertices are called the external vertices of the artery. A $k$-artery is an artery with exactly $k$ external vertices.

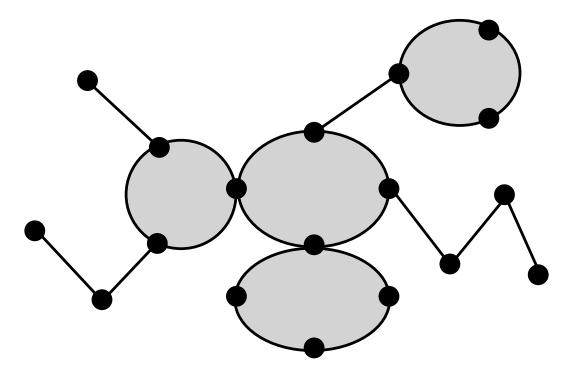

Figure 5: An artery.

The concept of an artery lies somewhere between a graphic tree and a path. An artery must also contain a unique a path between every pair of its vertices since it is 
incidence dual to a graphic tree, however, every internal vertex must also have degree equal to 2. The simplest arteries are a single vertex, which is a 1-artery or vertex-artery, and a graphic path, which is a 2 -artery.

Just as a graphical path can be thought of as a subdivision of a 2-edge, a $k$-artery can be regarded as a subdivision of a $k$-edge. The structure of arteries can be characterized through the operation of subdivision as indicated by the following useful lemmas.

Lemma 4.2.1. $A$ is a $k$-artery, $k \geqslant 2$, if, and only if, $A$ can be vertex-contracted into a $k$-edge.

Lemma 4.2.2. $A$ is a $k$-artery, $k \geqslant 2$, if, and only if, $A$ is a subdivision of a $k$-edge.

Flowers and pseudo-flowers are simplified in signed graphs, as indicated by Propositions 4.1.2 and 4.1.3, and we have the following result for arteries of signed graphs.

Proposition 4.2.3. $A$ is an artery of a signed graph if, and only if, $A$ is a path.

\subsection{Arterial Connections and Hypercircles}

Two hypergraphs that are either disjoint or have a single vertex in common are said to be nearly-disjoint. An arterial connection of hypergraphs is the union of a collection of pairwise nearly-disjoint hypergraphs $\mathcal{H}$ with a collection of pairwise disjoint arteries $\mathcal{A}$ satisfying:

AC1. The arterial connection is connected.

AC2. If $H \in \mathcal{H}, A \in \mathcal{A}$, and $H \cap A \neq \emptyset$, then $H \cap A=(v, \emptyset, \emptyset)$ and $v$ is an external vertex of $A$.

AC3. If $H_{1}, H_{2} \in \mathcal{H}$ and $H_{1} \cap H_{2} \neq \emptyset$, then $H_{1} \cap H_{2}=(w, \emptyset, \emptyset) \in \mathcal{A}$.

AC4. If $H_{1}, H_{2} \in \mathcal{H}$ and $H_{1} \cap H_{2}=(w, \emptyset, \emptyset)$, then $H_{1}$ and $H_{2}$ are the only elements of $\mathcal{H}$ that contain $w$.

AC5. Weak-deletion of any edge or vertex of an artery in $\mathcal{A}$ disconnects the arterial connection.

An arterial connection of special interest is a thorn-connection which is the union of a collection of nearly-disjoint pseudo-flowers $\mathcal{P}$ and a collection of pairwise disjoint arteries $\mathcal{A}$ satisfying:

TC1. A thorn-connection is an arterial connection.

TC2. If $P \in \mathcal{P}, A \in \mathcal{A}$, and $P \cap A \neq \emptyset$, then $P \cap A=(t, \emptyset, \emptyset)$ where $t$ is a thorn of $P$. 
Observe that if two pseudo-flowers of a thorn-connection have a vertex in common then it must be a thorn of each. An arterial connection is said to be floral if it contains no monovalent vertices.

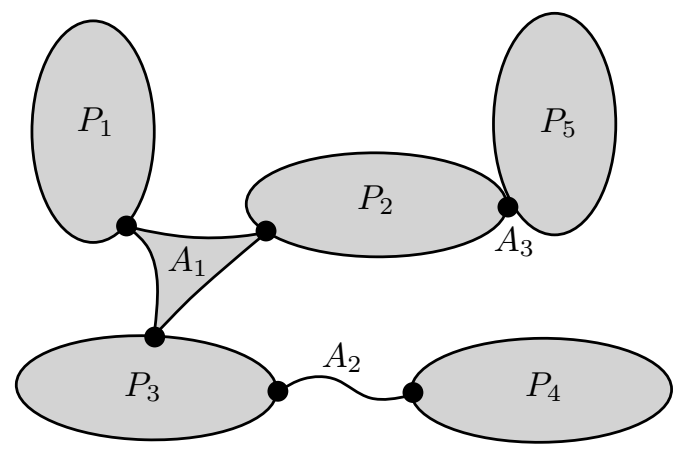

Figure 6: A floral thorn-connection with its pseudo-flowers and connecting arteries depicted by the labeled regions.

The oriented hypergraph resulting from the vertex-contraction of the vertices belonging to the arteries of a floral thorn-connection is a hypercircle. A hypercircle containing exactly $k \geqslant 2$ flower-parts is called a $k$-hypercircle, while a 0 -edge is a 0 -hypercircle, and flower is a 1-hypercircle.

We say two pseudo-flowers $P_{1}$ and $P_{2}$ are adjacent if they share a single edge which is also an isthmus in $P_{1} \cup P_{2}$. A hypercircle is the vertex-contraction of a floral thornconnection into adjacent pseudo-flowers.

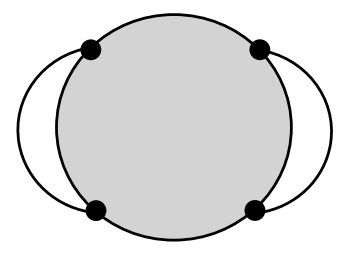

G

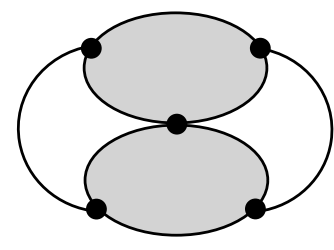

$G^{\prime}$

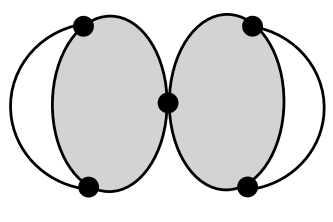

$G^{\prime \prime}$

Figure 7: A hypercircle $G$, a flower $G^{\prime}$ that vertex-contracts into $G$, and a floral thornconnection $G^{\prime \prime}$ that vertex-contracts into $G$

\section{The Cyclomatic Number and Balance}

\subsection{The Incidence Graph}

The oriented incidence graph of an oriented hypergraph $G=\left(V_{G}, E_{G}, \mathcal{I}_{G}, \sigma\right)$ is the oriented bipartite graph $\Gamma_{G}$ with vertex set $V_{\Gamma}=V_{G} \cup E_{G}$, edge set $E_{\Gamma}=\mathcal{I}_{G}$ and orientation function $\sigma$. Observe that $\Gamma_{G}$ contains no parallel edges if, and only if, $G$ is an incidencesimple hypergraph. 

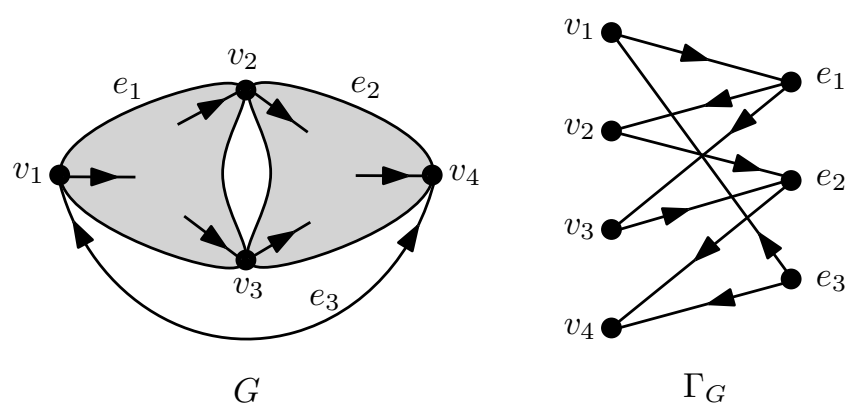

Figure 8: An oriented hypergraph $G$ and its incidence graph $\Gamma$.

Our focus is on extending column dependency results to oriented hypergraphic families using edge-induced oriented subhypergraphs. When viewed through bipartite graphs edgeinduced oriented hypergraphs are subgraphs induced by a subset of one of the vertex set bipartitions - whatever one was determined to be the "edges". Thus, any structural families would not be symmetric in general bipartite graphs. While incidence graphs are not suited to study the entire problem of dependencies, it does provide an alternate point of view to examine some oriented hypergraphic concepts.

Lemma 5.1.1. $C$ is a circle of an oriented hypergraph $G$ if, and only if, $C$ is a circle of the incidence graph $\Gamma$.

Proof. A hypergraphic circle is a sequence $a_{0}, i_{1}, a_{1}, i_{2}, a_{2}, i_{3}, a_{3}, \ldots, a_{2 k-1}, i_{2 k}, a_{2 k}$, where $\left\{a_{j}\right\}$ is an alternating sequence of vertices and edges, $i_{j}$ is an incidence containing $a_{j-1}$ and $a_{j}$, and no vertex, edge, or incidence is repeated except $a_{0}=a_{2 k}$. In the incidence graph this is an alternating sequence of vertices (hypergraph vertices and edges) and edges (hypergraph incidences) where no vertex or edge repeats and whose end-points coincide, which is a graphic circle. The definitions coincide when translating from hypergraphs to incidence graphs.

A chord of a graphic circle $C$ is an edge not in $C$ whose end-points are in $C$.

Lemma 5.1.2. $C$ is a degenerate circle of an oriented hypergraph $G$ if, and only if, there exists a chord of $C$ in the incidence graph $\Gamma$.

Proof. A circle $C$ is degenerate if there is an incidence that does not appear in the circle but belongs to an edge and vertex of the circle. In the incidence graph this incidence is an edge not in $C$ whose end-points are in $C$. The concepts coincide when translating between hypergraphs and incidence graphs.

We immediately have a restatement of Lemma 5.1.2 in terms of pure circles.

Corollary 5.1.3. $C$ is a pure circle of an oriented hypergraph $G$ if, and only if, $C$ is chord-free in the incidence graph $\Gamma$.

Some of the terms introduced for oriented hypergraphs are direct translations from graphic definitions when viewed through incidence graphs. 
Lemma 5.1.4. Let $G$ be an oriented hypergraph with incidence graph $\Gamma . G$ is inseparable if, and only if, $\Gamma$ is inseparable.

Proof. $G$ is inseparable if every pair of incidences is contained in a circle, while $\Gamma$ is inseparable if every pair of edges is contained in a circle. The edges of $\Gamma$ are the incidences of $G$.

Lemma 5.1.5. Let $G$ be an oriented hypergraph with incidence graph $\Gamma . G$ is incidencesimple if, and only if, $\Gamma$ is simple.

Proof. $G$ is incidence-simple if there are no multiple incidences, while $\Gamma$ is simple there are no parallel edges, as loops do not occur in bipartite graphs. The edges of $\Gamma$ are the incidences of $G$.

\subsection{The Cyclomatic Number for Oriented Hypergraphs}

Since circles of an oriented hypergraph are in one-to-one correspondence with the circles in its incidence graph the graphic cyclomatic number of the incidence graph can be regarded as the cyclomatic number of the oriented hypergraph. The graphic cyclomatic number $\varphi$ is equal to the number of edges that lie outside a maximal forest of a graph $\Gamma$ and is given by:

$$
\varphi_{\Gamma}=\left|E_{\Gamma}\right|-\left|V_{\Gamma}\right|+c,
$$

where $c$ is the number of connected components of $\Gamma$.

Using the graphic cyclomatic number for the bipartite incidence graph of an oriented hypergraph, where $V_{\Gamma}$ consists of the vertices and edges of $G$ and $E_{\Gamma}$ consists of the incidences of $G$, we define the cyclomatic number of an oriented hypergraph $G$ as

$$
\varphi_{G}:=\left|\mathcal{I}_{G}\right|-\left(\left|V_{G}\right|+\left|E_{G}\right|\right)+c,
$$

where $c$ is the number of connected components of $G$.

We also have the following alternate, incidence dual, ways of calculating the hypergraphic cyclomatic number which are consistent with Berge's formulation of the cyclomatic number in [2]:

$$
\varphi_{G}=\sum_{e \in E_{G}}|e|-\left(\left|V_{G}\right|+\left|E_{G}\right|\right)+c
$$

or

$$
\varphi_{G}=\sum_{v \in V_{G}}[\operatorname{deg}(v)]-\left(\left|V_{G}\right|+\left|E_{G}\right|\right)+c .
$$

Lemma 5.2.1. The hypergraphic cyclomatic number of a graph is equal to its graphic cyclomatic number.

Proof. In a graph $\Gamma$ every edge has size 2 so $|\mathcal{I}|=2|E|$. Replacing $|\mathcal{I}|$ in the hypergraphic cyclomatic number with $2|E|$ yields the result. 
Since the two cyclomatic numbers agree on graphs we will refer to a single cyclomatic number, the oriented hypergraphic version.

An alternate way to interpret the graphic cyclomatic number is that it is the minimal number of circles that must be "broken" in order to be left with an acyclic graph. While this is normally accomplished by deleting edges from the graph, in the incidence graph the edges are incidences of the corresponding oriented hypergraph so breaking a circle is the deletion of a single incidence of that circle. Specifically, deleting an incidence $i=(v, e, k, \sigma(v, e, k))$ from $G=(V, E, \mathcal{I}, \sigma)$ is the oriented hypergraph

$$
G \backslash i:=\left(V, E, \mathcal{I} \backslash i,\left.\sigma\right|_{(\mathcal{I} \backslash i)}\right)
$$

where $\mathcal{I} \backslash i$ determines both the incidence function and labelling sets for $G \backslash i$, while $\sigma$ is restricted to the new incidence set.

Corollary 5.2.2. If $G$ is a hypergraph, then the cyclomatic number $\varphi_{G}$ is the minimal number of circles of $G$ that need to be broken to yield an acyclic hypergraph.

A minimal collection of circles whose breaking leaves an acyclic hypergraph is called a collection of essential circles. The concept of an essential circle of a hypergraph is similar to that of a fundamental circle of a graph. A fundamental circle arises from the graphical property that a unique circle is created when introducing an edge outside of a spanning forest, while an essential circle is a hypergraphic property where a unique circle is created when introducing an incidence outside a hypergraph corresponding to a spanning forest in the incidence graph. We adopt the term "essential circle" as the incidence-centric oriented hypergraphic concept and reserve the word "fundamental" as an edge-centric concept. Specifically, the choice of terminology is motivated as to not create confusion with the matroid theoretic concept of a fundamental circuit.

Corollary 5.2.3. If $G$ is a hypergraph, then $\varphi_{G}$ is equal to the size of any collection of essential circles in $G$.

Lemma 5.2.4. If $G$ is an oriented hypergraph and $H$ a subdivision of $G$, then $\varphi_{G}=\varphi_{H}$.

Proof. Subdivision cannot create any new connected components and creates exactly one new edge, one new vertex, and two new incidences, producing a net change of 0 in the cyclomatic number.

Subdivision may create new circles but does not destroy existing circles, so subdividing may only create new collections of essential circles.

Corollary 5.2.5. Any collection of essential circles of an oriented hypergraph $G$ corresponds to a collection of essential circles in a subdivision $H$ of $G$. 


\subsection{Theta Graphs}

A theta graph is a set of three internally disjoint paths whose end-points coincide. A vertex-theta-graph is a theta graph whose end-points are vertices, an edge-theta-graph is a theta graph whose end-points are edges, and a cross-theta-graph is a theta graph whose end-points consist of one vertex and one edge.
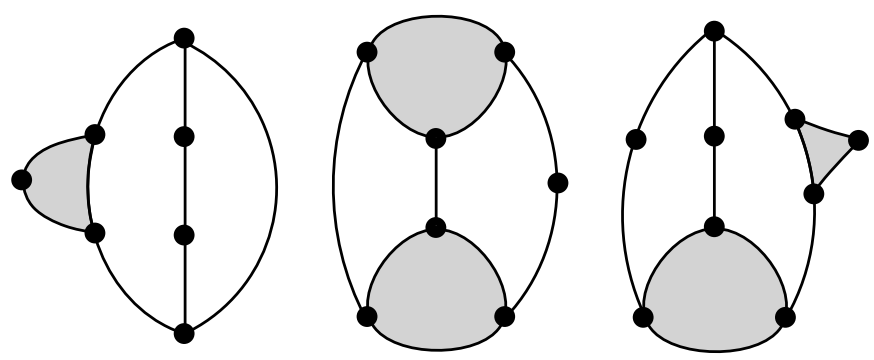

Figure 9: Hypergraphs that contain a vertex-theta, edge-theta, and cross-theta, respectively.

The paths of a theta graph form three internally disjoint paths in the incidence graph. The paths of a vertex-theta begin and end on the vertex side of the incidence graph, the paths of an edge-theta begin and end on the edge side of the incidence graph, and the paths of a cross-theta have one end-point on each side of the incidence graph. Since the incidence graph is bipartite the paths in vertex-thetas and edge-thetas must have even length in the incidence graph, while the paths of a cross-theta must have odd length in the incidence graph.

Lemma 5.3.1. If an oriented hypergraph contains an incidence of multiplicity $k \geqslant 3$, then it contains a cross-theta.

Proof. Any incidence with multiplicity $k \geqslant 3$ in an oriented hypergraph corresponds to $k$ parallel edges in the incidence graph. Any three of them correspond to a cross-theta in the oriented hypergraph.

Lemma 5.3.2. If an oriented hypergraph contains a degenerate circle, then it contains a cross-theta.

Corollary 5.3.3. Every circle in a cross-theta-free oriented hypergraph is pure.

Lemma 5.3.4. If $G$ contains a cross-theta, then the incidence dual $G^{*}$ contains a crosstheta.

Lemma 5.3.5. If $G$ contains a cross-theta, then any subdivision of $G$ contains a crosstheta.

While incidences of multiplicity 3 or greater contain cross-thetas, we may subdivide the oriented hypergraph to produce an incidence-simple, degenerate-circle-free, oriented hypergraph that necessarily contains a cross-theta. The relationship between cross-thetas, 
degenerate circles, and multiple incidences plays an essential role in extending the investigation of minimal column dependencies of $\{0, \pm 1\}$-matrices to determining minimal column dependencies of integral matrices.

We know from signed graphs that circles factor prominently into the classification of the minimal dependencies, but the sign of each circle plays an important part as well.

Lemma 5.3.6. A vertex-theta or an edge-theta contains an even number of negative circles.

Proof. We will show the result for vertex-thetas, and observe that incidence duality and Lemma 2.3.1 completes the proof for edge-thetas.

Let the paths connecting the end-points of a vertex-theta be $P_{1}, P_{2}$, and $P_{3}$. Let the signs of the three paths connecting the end-points be $\varepsilon_{1}, \varepsilon_{2}$, and $\varepsilon_{3}$ respectively.

From these three paths we have the following circles: $C_{1}=P_{1} \cup P_{2}, C_{2}=P_{1} \cup P_{3}$, and $C_{3}=P_{2} \cup P_{3}$. The sign of $C_{1}$ is $\varepsilon_{1} \varepsilon_{2}$, the sign of $C_{2}$ is $\varepsilon_{1} \varepsilon_{3}$, and the sign of $C_{3}$ is $\varepsilon_{2} \varepsilon_{3}$. If the $\varepsilon_{i}$ are all + or all - then each circle is positive, thus there are no negative circles. If the $\varepsilon_{i}$ do not all have the same sign then without loss of generality suppose $\varepsilon_{1}=+$ and $\varepsilon_{2}=-$. Then the sign of $C_{1}$ is negative, and depending on $\varepsilon_{3}$ exactly one of $C_{2}$ or $C_{3}$ will be negative as well.

Lemma 5.3.7. A cross-theta contains an odd number of negative circles.

Proof. Let the end-points of the three cross-paths in a cross-theta be vertex $v$ and edge $e$. Let the cross-paths be $P_{1}, P_{2}$, and $P_{3}$ and the vertices of $e$ that belong to these cross-paths be $v_{1}, v_{2}$, and $v_{3}$, respectively. Let $C_{i j}$ be the circle formed by paths $P_{i}$ and $P_{j}$ along with edge $e$.

Case 1a: Suppose $\operatorname{sgn}\left(P_{1}\right)=\operatorname{sgn}\left(P_{2}\right)=\operatorname{sgn}\left(P_{3}\right)$, and $\sigma\left(v_{1}, e\right)=\sigma\left(v_{2}, e\right)=\sigma\left(v_{3}, e\right)$. Then $\operatorname{sgn}\left(C_{12}\right)=\operatorname{sgn}\left(C_{13}\right)=\operatorname{sgn}\left(C_{23}\right)=-1$.

Case 1b: Suppose $\operatorname{sgn}\left(P_{1}\right)=\operatorname{sgn}\left(P_{2}\right)=\operatorname{sgn}\left(P_{3}\right)$, and $\sigma\left(v_{1}, e\right)=\sigma\left(v_{2}, e\right) \neq \sigma\left(v_{3}, e\right)$. Then $\operatorname{sgn}\left(C_{12}\right)=-1$, and $\operatorname{sgn}\left(C_{13}\right)=\operatorname{sgn}\left(C_{23}\right)=+1$.

Case 2a: Suppose $\operatorname{sgn}\left(P_{1}\right)=\operatorname{sgn}\left(P_{2}\right) \neq \operatorname{sgn}\left(P_{3}\right)$, and $\sigma\left(v_{1}, e\right)=\sigma\left(v_{2}, e\right)=\sigma\left(v_{3}, e\right)$. Then $\operatorname{sgn}\left(C_{12}\right)=-1$, and $\operatorname{sgn}\left(C_{13}\right)=\operatorname{sgn}\left(C_{23}\right)=+1$.

Case 2b: Suppose $\operatorname{sgn}\left(P_{1}\right)=\operatorname{sgn}\left(P_{2}\right) \neq \operatorname{sgn}\left(P_{3}\right)$, and $\sigma\left(v_{1}, e\right)=\sigma\left(v_{2}, e\right) \neq \sigma\left(v_{3}, e\right)$. Then $\operatorname{sgn}\left(C_{12}\right)=-1$, and $\operatorname{sgn}\left(C_{13}\right)=\operatorname{sgn}\left(C_{23}\right)=+1$.

Case 2c: Suppose $\operatorname{sgn}\left(P_{1}\right)=\operatorname{sgn}\left(P_{2}\right) \neq \operatorname{sgn}\left(P_{3}\right)$, and $\sigma\left(v_{1}, e\right) \neq \sigma\left(v_{2}, e\right)=\sigma\left(v_{3}, e\right)$. Then $\operatorname{sgn}\left(C_{13}\right)=-1$, and $\operatorname{sgn}\left(C_{12}\right)=\operatorname{sgn}\left(C_{23}\right)=+1$.

Up to relabeling, these cases exhaust all possible combinations of path signs and incidence signs in a cross-theta. In every case there are an odd number of negative circles.

While a cross-theta presents a negative circle problem unique to hypergraphs, the following theorem examines the structural properties of cross-thetas in flowers.

Theorem 5.3.8. If a flower contains a vertex of degree $\geqslant 3$, then it contains a cross-theta. 
Proof. By subdividing out all degenerate circles and all multiple incidences we only need to consider incidence-simple, degenerate-circle-free, flowers since subdivision does not remove cross-thetas by Lemma 5.3.5.

Let $F$ be an incidence-simple, degenerate-circle-free, flower containing a vertex $v$ such that $\operatorname{deg}(v) \geqslant 3$, and let three of the edges incident to $v$ be $e_{1}, e_{2}$, and $e_{3}$. Since $F$ is a flower we know that there must be a circle $C$ containing the incidences $\left(v, e_{1}\right)$ and $\left(v, e_{2}\right)$. Also, since $F$ is degenerate-circle-free, $e_{3}$ cannot belong to $C$. $C$ must contain an edge of size $\geqslant 3$ in $F$ or there would be a smaller flower, namely the circle-hypergraph corresponding to $C$, contradicting minimality of $F$.

Let $\mathcal{P}$ be the collection of paths in $F$ containing the incidence $\left(v, e_{3}\right)$ with one endpoint vertex $v$ and the other an element of $C$ such that each path is internally disjoint from $C$. The elements of $\mathcal{P}$ are either cross-paths or vertex-paths, depending on non- $v$ end-point of $C$. $\mathcal{P}$ must contain at least one cross-path or else every path of $\mathcal{P}$ would be a vertex-path and the hypergraph resulting from the deletion of all the non-end-point elements of the paths of $\mathcal{P}$ would result in a smaller flower, contradicting the minimality of $F$.

Let $Q$ be a shortest cross-path of $\mathcal{P}$, and let the end-points of $Q$ be $v$ and $e$. Regard $C$ as two internally disjoint cross-paths $P_{1}$ and $P_{2}$ each with end-points $v$ and $e$ as well, this can be done since $e$ must also be an element of $C$. $P_{1}, P_{2}$, and $Q$ are three internally disjoint cross-paths whose end-points coincide, and $F$ contains a cross-theta.

From this result we have the following corollary concerning cross-theta-free flowers.

Corollary 5.3.9. Every vertex of a cross-theta-free flower must have degree equal to 2.

Proof. We know from Theorem 5.3.8 if a flower has a vertex of degree $\geqslant 3$ it must contain a cross-theta, so the degree of every vertex in a cross-theta-free flower must be $\leqslant 2$. However, a flower cannot contain a monovalent vertex or an isolated vertex since it would not be inseparable. Thus the degree of every vertex in a cross-theta-free flower must be exactly 2.

\subsection{Ear Decompositions of Flowers}

Let $H$ be a hypergraph, $P$ be a path containing at least one incidence, and $G(P)$ be the path-hypergraph corresponding to the elements of $P$. The hypergraph $H \cup G(P)$ is said to result from adjoining an ear to $H$ if $H \cap G(P)$ consists of only the end-points of $P$. This concept of adjoining an ear follows the development in [5]. When viewing this process from the incidence graph, adjoining an ear to a hypergraph $H$ is equivalent to the graphical concept of adjoining an ear in the incidence graph $\Gamma_{H}$.

Adjoining an ear to a bipartite graph either connects the vertices within a single part of the bipartition or connects the vertices across the bipartition with a path. The connecting path is a vertex-path or an edge-path in the corresponding oriented hypergraph if the endpoints lie in a single part of the bipartition, and is a cross-path if the end-points lie in different parts of the bipartition. Observe that any path that connects to the edge-part 
of the bipartition would increase the size of the edge in the oriented hypergraph. A hypergraph that can be constructed starting from a single vertex or edge by sequentially adjoining ears is said to have an ear decomposition.

The following is a known result (see [5]) concerning the structure of graphs.

Theorem 5.4.1. A connected graph has an ear decomposition if, and only if, it is inseparable.

We are especially interested in the structure of cross-theta-free flowers and applying this result to the incidence graph of a flower.

Theorem 5.4.2. If $F$ is a cross-theta-free flower, then every ear decomposition of $F$ can be regarded as consisting of only edge-paths.

Proof. Let $\Gamma$ be the incidence graph of $F$ with $V_{\Gamma}=V_{F} \cup E_{F}$. From Lemma 5.1.4 we know that $\Gamma$ is inseparable since $F$ is a flower. Given an ear decomposition of $\Gamma$ regard the first circle as adjoining an ear to a vertex of $E_{F}$. This can be done because $\Gamma$ is bipartite.

By Theorem 5.3.8 we know that $F$ cannot contain a vertex of degree 3 or greater, so adjoining additional ears in $\Gamma$ must connect two vertices in $E_{F}$, the edge-vertices of the bipartition, or else we would have a degree-3 vertex.

Corollary 5.4.3. Let $\mathcal{P}$ be a collection of paths of an ear decomposition of a cross-thetafree flower $F$. Every path of $\mathcal{P}$ must contain a unique vertex that does not belong to any other path of $\mathcal{P}$.

Proof. Using Theorem 5.4.2 we see that any path in an ear decomposition of $\Gamma_{F}$ must connect two vertices of $\Gamma_{F}$ that are edges of $F$. Since $\Gamma_{F}$ is bipartite every such path must have even length, and every path must contain a unique vertex of $\Gamma_{F}$ that corresponds to a vertex of $F$.

This provides us with the following property for a collection of essential circles of a cross-theta-free oriented hypergraph.

Corollary 5.4.4. Given a collection of essential circles of a cross-theta-free flower $F$, there exists a set of distinct vertex representatives for each essential circle.

Proof. Let $F$ be a cross-theta-free flower and $\mathcal{C}$ be a set of essential circles of $F$. Since $F$ is inseparable, by Theorem 5.4.2, $F$ can be built by adjoining edge-path ears. Moreover, a collection of essential circles has a natural ear decomposition since they generate all the circles of $F$ and the circles of $F$ and $\Gamma_{F}$ are in 1-1 correspondance. By Corollary 5.4.3 there must exist a vertex in each essential circle that does not belong to any other circle in $\mathcal{C}$. 


\subsection{Balance in Oriented Hypergraphs}

An oriented hypergraph is balanced if all circles are positive and balanceable if there are incidences that can be negated so that the resulting oriented hypergraph is balanced. An oriented hypergraph that is not balanceable is said to be unbalanceable. Clearly, any oriented hypergraph containing a cross-theta must necessarily be unbalanceable by Theorem 5.3.7.

The concept of a balanced non-oriented hypergraph was introduced by Berge in [1] as one of a number of different generalizations of bipartite graphs. Berge defined a hypergraph as balanced if every odd circle has an edge containing three vertices of the circle. Such a hypergraph clearly contains a cross-theta, additionally, Berge used $\{0,1\}$-incidence matrices thus forcing odd circles to be negative.

Balanced $\{0, \pm 1\}$-matrices were introduced by Truemper in [13] as a generalization of a balanced hypergraph. Truemper exhibited two minimal obstructions for balanceability in [14] using bipartite incidence graphs - odd-spoked wheels and 3 internally disjoint odd paths - both of which contain cross-thetas.

Theorem 5.5.1. A bipartite graph is balanceable if, and only if, it does not contain an odd wheel or a 3-odd-path configuration as a subgraph.

As immediate corollaries we have:

Theorem 5.5.2. An oriented hypergraph $G$ is balanceable if, and only if, it does not contain a cross-theta.

Corollary 5.5.3. The multiplicity of any incidence in a balanceable oriented hypergraph is at most 2.

Thus the concept of balanceability in an oriented hypergraph is a weakening of the concept of a balanceable matrix, specifically, a matrix is balanced if all pure circles are positive, while an oriented hypergraph is balanced if all circles are positive. A survey of balanced matrices by M. Conforti, G. Cornuéjols, and K. Vuškovic can be found in [4].

\section{Circuit Classification of Balanced Oriented Hyper- graphs}

\subsection{Balanced Flowers}

The classification of the minimal dependencies of graphs is a well known result.

Theorem 6.1.1. The minimal dependencies of a graph are circles.

Using Proposition 4.1.2, we can translate Theorem 6.1.1 using oriented hypergraphic terminology that is indicative of the dependency results we will obtain for balanced oriented hypergraphs. 
Theorem 6.1.2. The minimal dependencies of a graph are balanced flowers.

The focus in this section is on the extension of Theorem 6.1.1 to oriented hypergraphs by examining balanced flowers.

Lemma 6.1.3. A balanced flower does not contain a vertex of degree $\geqslant 3$.

Proof. If a balanced flower had a vertex of degree $\geqslant 3$ then by Theorem 5.3.8 it would contain a cross-theta, and by Lemma 5.3 .7 would contain a negative circle.

Lemma 6.1.3 incorporates Theorem 5.3.8 into the theory of balanced oriented hypergraphs, and has an immediate result paralleling Corollary 5.3.9.

Lemma 6.1.4. The degree of every vertex in a balanced flower must be 2 .

Proof. From Corollary 5.3.9 we know that every vertex of a cross-theta-free flower must have degree equal to 2. A balanced flower is necessarily cross-theta-free.

Observe that Lemma 6.1.4 implies that the incidence dual of a balanced flower is a signed graph.

Finally, we arrive at our first family of minimally dependent oriented hypergraphs.

Theorem 6.1.5. A balanced flower is minimally dependent.

Proof. Let $F$ be a balanced flower, and observe that a flower cannot contain a 1-edge.

Case 1: If $F$ is a 0-edge, then it corresponds to a single column of 0 's and is minimally dependent.

Case 2: If $F$ consists of only edges of size 2, then it is already minimally dependent as it is a positive signed graphic circle.

Case 3: If $F$ contains an edge of size 3 or greater, then we will show that it is minimally dependent.

Let $\mathrm{H}_{F}$ be the incidence matrix of $F$ and let $\Gamma_{F}$ be the incidence graph of $F$. Take a spanning tree of $\Gamma_{F}$ to determine a collection of essential circles of $F$ by translating the fundamental circles of $\Gamma_{F}$ to $F$. The sign of each essential circle is + since $F$ is balanced.

Since each essential circle is pure and positive we can take a linear combination of the rows corresponding to the vertices of that circle to zero out any row in the square submatrix corresponding to the vertices and edges of the circle. Moreover, we know that every vertex has degree 2 by Lemma 6.1.4, thus there are no other non-zero entries in the rows of $\mathrm{H}_{F}$ outside of the square submatrix corresponding to the circle, and the entire row in $\mathrm{H}_{F}$ must be zero after row reducing.

Corollary 5.4.4 tells us there is a unique vertex for each essential circle that does not belong to any other essential circle in the given collection. For each essential circle take a linear combination of the rows corresponding to the vertices of that circle so that a row corresponding to a vertex unique to that essential circle is zero. Since this vertex is not contained in any other essential circles we can zero out a row for each essential circle. Thus we can zero out exactly $\varphi_{F}$ rows since the essential circles of $F$ are fundamental circles of $\Gamma_{F}$. Hence, the row rank of $\mathrm{H}_{F}$ is $\left|V_{F}\right|-\varphi_{F}$. 
In order to complete the proof we must show that $\mathrm{H}_{F}$ has nullity 1 . Since we know the row rank is $\left|V_{F}\right|-\varphi_{F}$ we must show

$$
\left|V_{F}\right|-\varphi_{F}=\left|E_{F}\right|-1
$$

Solving for $\varphi_{F}$ this is equivalent to showing

$$
\varphi_{F}=\left|V_{F}\right|-\left|E_{F}\right|+1
$$

By the definition of the cyclomatic number we have

$$
\varphi_{F}=\left|\mathcal{I}_{F}\right|-\left(\left|V_{F}\right|+\left|E_{F}\right|\right)+1 \text {. }
$$

However, since the degree of every vertex of $F$ is equal to 2 we have $\left|\mathcal{I}_{F}\right|=2\left|V_{F}\right|$. Replacing this into the cyclomatic number we get

$$
\begin{aligned}
\varphi_{F} & =\left|\mathcal{I}_{F}\right|-\left(\left|V_{F}\right|+\left|E_{F}\right|\right)+1 \\
& =2\left|V_{F}\right|-\left(\left|V_{F}\right|+\left|E_{F}\right|\right)+1 \\
& =\left|V_{F}\right|-\left|E_{F}\right|+1 .
\end{aligned}
$$

Solving this for $\left|V_{F}\right|-\varphi_{F}$ we get

$$
\left|V_{F}\right|-\varphi_{F}=\left|E_{F}\right|-1
$$

and the nullity of $\mathrm{H}_{F}$ is equal to 1 .

Note that Theorem 6.1 .5 can be proved using signed graph theory since the incidence dual of a balanced oriented hypergraph is a signed graph.

\subsection{Balanced Pseudo-Flowers}

There is only a single signed graphic example of a balanced minimal dependency involving pseudo-flowers: two 1-edges connected by a path of length $\geqslant 0$. Each 1-edge is a pseudoflower whose flower-part is the 0-edge resulting from weak deletion of the vertex. Oriented hypergraphs, however, can have more pseudo-flowers than just 1-edge pseudo-flowers.

Lemma 6.2.1. Let $P_{1}, P_{2}, \ldots, P_{k}$ be a collection of disjoint balanced 1-pseudo-flowers, and e a $k$-edge that meets only the thorn of each $P_{i}$. The oriented hypergraph $G:=P_{1} \cup P_{2}$ $\cup \ldots \cup P_{k} \cup$ e is minimally dependent.

Proof. Each pseudo-flower $P_{i}$ contains a balanced flower-part, thus the degree of every vertex must be equal to 2 . For each $P_{i}$ there are $\varphi_{P_{i}}$ essential circles so there are $\varphi_{P_{i}}$ rows that can be zeroed out since the flower-part is balanced, and must contain only vertices of degree equal to 2 . 
Since there are no circles in $G$ other than those in the flower parts of each $P_{i}$ we have

$$
\begin{aligned}
\varphi_{G} & =\sum_{i=1}^{k} \varphi_{P_{i}} \\
& =\left|\mathcal{I}_{G}\right|-\left(\left|V_{G}\right|+\left|E_{G}\right|\right)+1 .
\end{aligned}
$$

Since the degree of every vertex in $G$ is 2 we have $\left|\mathcal{I}_{G}\right|=2\left|V_{G}\right|$. Substituting into $\varphi_{G}$ we get

$$
\begin{aligned}
\varphi_{G} & =\left|\mathcal{I}_{G}\right|-\left(\left|V_{G}\right|+\left|E_{G}\right|\right)+1 \\
& =2\left|V_{G}\right|-\left(\left|V_{G}\right|+\left|E_{G}\right|\right)+1 \\
& =\left|V_{G}\right|-\left|E_{G}\right|+1 .
\end{aligned}
$$

Solving for $\left|V_{G}\right|-\varphi_{F}$ we get

$$
\left|V_{G}\right|-\varphi_{F}=\left|E_{G}\right|-1 \text {. }
$$

That is, the row rank of the incidence matrix of $G$ is $\left|E_{G}\right|-1$, so $G$ is dependent with nullity equal to 1 .

To see that $G$ is minimally dependent observe that the weak deletion of any nonempty subset of edges would either disconnect $G$ or result in a monovalent vertex, and is not minimally dependent. Since no edge-induced subhypergraph is of $G$ is minimally dependent, $G$ must be minimally dependent.

The thorns of each pseudo-flower in Lemma 6.2.1 can be switched so that every thorn is compatibly oriented with respect to edge $e$. Vertex-contracting these thorns produces a collection of $k$ adjacent pseudo-flowers sharing a single common isthmus. Moreover, this is obtained by operations that do not alter minimal dependencies, giving us the following corollary:

Corollary 6.2.2. A balanced hypercircle with a single isthmus is minimally dependent.

A cautionary note concerning Corollary 6.2.2: vertex-contracting a thorn-connection into a hypercircle may not be possible in the larger ambient oriented hypergraph, so any comparisons between thorn-connections and hypercircles must be done on the edgeinduced subhypergraph in order to examine the structure of minimal dependencies (i.e., we restrict to a specific set of columns when searching for dependency.)

It is important to point out that the subdivision of an isthmus in a balanced hypercircle need not be compatible to preserve minimal dependency since any newly created vertex will not belong to any circle in the subdivision. The subdivision could, however, alter another minimal dependency in a larger ambient oriented hypergraph in which the subdivided edge belongs to a circle. Thus, we say a subdivision of $G$ is balanced if the subdivision is compatible, or the subdivision is incompatible and the newly created vertex does not belong to a circle in the subdivision of $G$.

Lemma 6.2.3. A subdivision $H$ of $G$ is balanced if, and only if, the circles of $H$ corresponding to circles of $G$ have the same sign in both $G$ and $H$. 
Lemma 6.2.4. If $G$ is minimally dependent, then any balanced subdivision of $G$ is minimally dependent.

Proof. Let $H$ be a balanced subdivision of $G$. Call the subdivided edge $e$ and the new edges resulting from the subdivision $e_{1}$ and $e_{2}$.

If the balanced subdivision is compatible then, from Corollary 3.2.4, we know that a compatible subdivision of a minimal dependency is still minimally dependent.

If the balanced subdivision is incompatible then the newly created vertex in the subdivision does not belong to any circle of $H$. Since the new vertex does not belong to any circle of $H$ it does not increase the cyclomatic number or the nullity, but does increase the number of vertices and edges each by 1 , so $H$ must be dependent.

To see that $H$ is minimally dependent observe that both $H \backslash e_{1}$ and $H \backslash e_{2}$ contains a monovalent vertex, and by Lemma 2.2.1, neither can be minimally dependent. Moreover, weak deletion of any other edge is equivalent to weak deletion is $G$, which is already minimally dependent. So no proper edge-induced subhypergraph of $H$ is minimally dependent, but $H$ is dependent, so $H$ is minimally dependent.

Corollary 6.2.5. A floral thorn-connection of $k$ balanced 1-pseudo-flowers is minimally dependent.

Building on this we have the following lemma concerning balanced hypercircles:

Theorem 6.2.6. A balanced hypercircle is minimally dependent.

Proof. Let $\mathcal{H}$ be a balanced hypercircle with maximal pseudo-flowers $P_{1}, P_{2}, \ldots, P_{k}$, whose respective flower-parts are $F_{1}, F_{2}, \ldots, F_{k}$. Since their flower-parts are pairwise disjoint and $\mathcal{H}$ is balanced, every vertex belonging to some flower-part must have degree equal to 2. Also note that every thorn of a pseudo-flower belongs to the flower-part of another adjacent pseudo-flower, so all the vertices of $\mathcal{H}$ must have degree equal 2 , thus giving $\left|\mathcal{I}_{\mathcal{H}}\right|=2\left|V_{\mathcal{H}}\right|$.

Observe that any collection of essential circles of $\mathcal{H}$ is the union of a collection of essential circles for each flower-part since the flower-parts are pairwise disjoint, and there are no circles outside of the flower-parts. Thus we have that

$$
\varphi_{\mathcal{H}}=\sum_{i=1}^{k} \varphi_{P_{i}}=\sum_{i=1}^{k} \varphi_{F_{i}}
$$

For each flower-part we are able to zero out $\varphi_{F_{i}}$ rows for a total of $\varphi_{\mathcal{H}}$ zero-rows. However,

$$
\varphi_{\mathcal{H}}=\left|\mathcal{I}_{\mathcal{H}}\right|-\left(\left|V_{\mathcal{H}}\right|+\left|E_{\mathcal{H}}\right|\right)+1
$$

and substituting $\left|\mathcal{I}_{\mathcal{H}}\right|=2\left|V_{\mathcal{H}}\right|$ we have

$$
\begin{aligned}
\varphi_{\mathcal{H}} & =2\left|V_{\mathcal{H}}\right|-\left(\left|V_{\mathcal{H}}\right|+\left|E_{\mathcal{H}}\right|\right)+1 \\
& =\left|V_{\mathcal{H}}\right|-\left|E_{\mathcal{H}}\right|+1 .
\end{aligned}
$$


Solving this for $\left|V_{\mathcal{H}}\right|-\varphi_{\mathcal{H}}$ we see that

$$
\left|V_{\mathcal{H}}\right|-\varphi_{\mathcal{H}}=\left|E_{\mathcal{H}}\right|-1
$$

Thus the row rank is one less than the number of columns, and the incidence matrix has nullity equal to 1 . The weak deletion of any non-empty set of edges leaves a monovalent vertex, which is not minimally dependent by Lemma 2.2.1. So no edge-induced subhypergraph of $\mathcal{H}$ is minimally dependent, and $\mathcal{H}$ is dependent, so $\mathcal{H}$ is minimally dependent.

Since flowers are 1-hypercircles, and 0-edges are 0-hypercircles, we can regard every balanced minimal dependency discussed so far as subdivisions of balanced hypercircles. These, in fact, are the only balanced minimal dependencies.

Theorem 6.2.7. $G$ is a balanced minimal dependency if, and only if, $G$ is a balanced subdivision of a balanced hypercircle.

Proof. Theorem 6.2.6 and Lemma 6.2.4 tells us that a balanced subdivision of a balanced hypercircle is minimally dependent, so all that is left to see is the converse.

To see the converse let $G$ be a balanced minimal dependency, and observe that $G$ is a connected oriented hypergraph that cannot contain any vertices of degree equal to 0 or 1 , by Lemma 2.2.1. Since the degree of any vertex in a minimal dependency is at least $2, G$ must be a 0-edge, a floral thorn-connection of 1-edge pseudo-flowers, or it must contain a circle. Clearly, a 0-edge is minimally dependent, and is a 0-hypercircle by definition, while a floral thorn-connections of 1-edge pseudo-flowers are minimal dependencies by Corollary 6.2.5, so we must show that if $G$ contains a circle, then it is a balanced subdivision of a hypercircle.

Suppose $G$ is a balanced minimal dependency that contains a circle. Since $G$ contains a circle it must contain some circle belonging to a flower or flower-part of a pseudo-flower. If $G$ is a flower, then it is minimally dependent by Theorem 6.1.5. Moreover, $G$ cannot properly contain a flower without violating minimality of the dependency.

If $G$ contains a circle and is not a flower, then $G$ must contain a maximal pseudo-flower $P_{0}$. Since $G$ is balanced, every vertex in the flower-part of $P_{0}$ must have degree equal to 2 in $P_{0}$, by Lemma 6.1.4. Moreover, the thorns of $P_{0}$ must connect to the rest of $G$ via some edge in $G \backslash P_{0}$. Since there are no monovalent vertices in $G$, the paths leading from the thorns of $P_{0}$ into $G \backslash P_{0}$ must reach a circle or terminate at a 1-edge. If there are no other circles, then $G$ consists of a single pseudo-flower $P_{0}$ and disjoint paths leaving each thorn that meet 1-edge pseudo-flowers. Observe that the paths leaving the thorns of $P_{0}$ must begin with an end-point of an artery or else it violates minimality of the dependency.

If there is a circle of $G$ not in $P_{0}$, then there must exist a circle whose distance from $P_{0}$ is minimal. Let $P_{1}$ be a maximal pseudo-flower containing a nearest circle. Observe that $P_{0}$ and $P_{1}$ are flower-part-disjoint or there would exist a circle containing elements from each flower-parts, producing a larger pseudo-flower and contradicting maximality of the pseudo-flowers. Thus, there is a single path between $P_{0}$ and $P_{1}$; it is possible that $P_{0}$ and $P_{1}$ are adjacent. Let $A_{1}$ be the largest artery containing the path between $P_{0}$ and 
$P_{1}$ that avoids all circles of $G$. If all the circles of $G$ belong to the flower-parts of $P_{0}$ and $P_{1}$, then $P_{0} \cup P_{1} \cup A_{1}$ must connect to 1-edge pseudo-flowers at the unused end-points of $A_{1}$, producing a minimal dependency.

If there is a circle of $G$ that does not belong to $P_{0} \cup P_{1} \cup A_{1}$, there must exist another maximal pseudo-flower $P_{2}$ flower-part disjoint from $P_{0}$ and $P_{1}$ whose distance from $P_{0} \cup$ $P_{1} \cup A_{1}$ is minimal. Let $A_{2}$ be the largest artery containing the path between $P_{2}$ and $P_{0} \cup P_{1} \cup A_{1}$ that internally avoids $P_{2}$ and $P_{0} \cup P_{1} \cup A_{1}$ and all circles of $G$. If all the circles of $G$ belong to the flower-parts of $P_{0}, P_{1}$, and $P_{2}$, then $P_{0} \cup\left(P_{1} \cup A_{1}\right) \cup\left(P_{2} \cup A_{2}\right)$ must connect to 1-edge pseudo-flowers at the unused end-points of $A_{1}$ and $A_{2}$, producing a minimal dependency.

If there is a circle of $G$ does not belong to $P_{0} \cup\left(P_{1} \cup A_{1}\right) \cup\left(P_{2} \cup A_{2}\right)$ we inductively add maximal pseudo-flowers and arteries nearest to, and avoiding, the previous collection except with the possibility of monovalent vertices. We form the union

$$
P_{0} \cup\left(P_{1} \cup A_{1}\right) \cup \ldots \cup\left(P_{k} \cup A_{k}\right)
$$

until all circles are exhausted. Since there are no circles outside the pseudo-flowers, the remaining monovalent vertices must be incident to a single 1-edge to form the minimal dependency. This forces $G$ to be a subdivision of a balanced hypercircle.

\section{Acknowledgements}

Thanks to Thomas Zaslavsky and Gerard Cornuéjols for their feedback, and the anonymous referee whose suggestions greatly improved the presentation.

\section{References}

[1] C. Berge. Sur certains hypergraphes généralisant les graphes bipartites. In Combinatorial theory and its applications, I (Proc. Colloq., Balatonfüred, 1969), pages 119-133. North-Holland, Amsterdam, 1970.

[2] Claude Berge. Hypergraphs, volume 45 of North-Holland Mathematical Library. North-Holland Publishing Co., Amsterdam, 1989. Combinatorics of finite sets, Translated from the French.

[3] M. Conforti, G. Cornuéjols, and M.R. Rao. Decomposition of balanced matrices. J. Combin. Theory B, 77:292-406, 1999.

[4] Michele Conforti, Gérard Cornuéjols, and Kristina Vušković. Balanced matrices. Discrete Math., 306(19-20):2411-2437, 2006.

[5] Chris Godsil and Gordon Royle. Algebraic graph theory, volume 207 of Graduate Texts in Mathematics. Springer-Verlag, New York, 2001.

[6] F. Harary. On the notion of balance of a signed graphs. Michigan Math. J., 2:143-146, 1953-1954.

[7] James Oxley. Matroid Theory, Oxford Univ. Press, Oxford, 1992. 
[8] N. Reff and L.J. Rusnak. An oriented hypergraphic approach to algebraic graph theory. Linear Algebra Appl., 437(9):2262-2270, 2012.

[9] L. Rusnak. Oriented Hypergraphs, PhD thesis, Binghamton University, 2010.

[10] A. Schrijver. Combinatorial Optimization: Polyhedra and Efficiency, Springer, Berlin, 2003.

[11] C.-J. Shi and J. A. Brzozowski. A characterization of signed hypergraphs and its applications to VLSI via minimization and logic synthesis. Discrete Appl. Math., 90(1-3):223-243, 1999.

[12] Chuan-Jin Shi. A signed hypergraph model of the constrained via minimization problem. Microelectronics Journal, 23(7):533-542, 1992.

[13] K. Truemper. Alpha-balanced graphs and matrices and GF(3)-representability of matroids. J. Combin. Theory Ser. B, 32(2):112-139, 1982.

[14] Klaus Truemper. Effective logic computation, A Wiley-Interscience Publication. John Wiley \& Sons Inc., New York, 1998.

[15] Thomas Zaslavsky. Signed graphs. Discrete Appl. Math., 4(1):47-74, 1982. MR 84e:05095a. Erratum, ibid., 5 (1983), 248. MR 84e:05095b.

[16] Thomas Zaslavsky. Biased graphs. I. Bias, balance, and gains. J. Combin. Theory Ser. B, 47(1):32-52, 1989.

[17] Thomas Zaslavsky. Orientation of signed graphs. European J. Combin., 12(4):361$375,1991$. 